\title{
Erbium-Doped Fiber Amplification Assisted Multi-Wavelength Brillouin-Raman Fiber Laser
}

\author{
Aiman Ismail ${ }^{1,2 *}$, Hazwani Mohammad Helmi ${ }^{2}$, Md Zaini Jamaludin ${ }^{1,2}$, Fairuz Abdullah ${ }^{1,2}$, Abdul Hadi Sulaiman ${ }^{1}$, \\ Ker Pin Jern ${ }^{1,2}$ \\ ${ }^{I}$ Institute of Power Engineering, Universiti Tenaga Nasional, Malaysia \\ ${ }^{2}$ Department of Electronics and Communication Engineering, College of Engineering, Universiti Tenaga Nasional, Malaysia \\ *Corresponding author E-mail:aiman@uniten.edu.my
}

\begin{abstract}
Multi-wavelength fiber laser based on Brillouin scattering in optical fiber has the potential of application in dense wavelength division multiplexing (DWDM) system. To enhance the performance of the fiber lasers, researchers proposed usages of erbium, or Raman amplification techniques. In an earlier work, it was reported that extracting residual Raman pump out of the laser cavity improves the performance of a multi-wavelength Raman fiber laser. In this paper, we proposed a setup utilizing the residual Raman pump to pump an erbium-doped fiber in multi-wavelength Brillouin-Raman fiber laser. Results show that the additional erbium-doped fiber is capable of amplifying the propagating Brillouin Stokes by more than 15-dB. This in turn helps in achieving lower stimulated Brillouin threshold and subsequently allow for higher number of Brillouin Stokes lines to be generated.
\end{abstract}

Keywords: Brillouin Scattering; erbium-doped fiber amplifier; Multi-wavelength fiber laser; Raman amplifier.

\section{Introduction}

Multi-wavelength fiber laser generation from a single source has attracted many researchers in the past decades due to its potential applications in several fields [1-4]. There are several techniques applied to achieve multi-wavelength generations such as using Sagnac loop filters [5,6], array waveguide gratings [7], MachZehnder interferometer [8] etc. However, due to its design simplicity and narrow linewidth output, stimulated Brillouin scattering (SBS) based multi-wavelength generations gains the most attention $[9,10]$.

Multi-wavelength Brillouin fiber laser (MBFL) exploits nonlinear Brillouin scattering phenomenon in fiber optic to generate multiple signals using a single seed signal. The principle behind MBFL can be explained with the help of Fig. 1. A Brillouin pump (BP) as seed signal is injected into the laser cavity via a circulator. The $\mathrm{BP}$ then propagates towards a Brillouin gain medium (typically a single mode fiber). If the BP power exceeds the stimulated Brillouin scattering threshold (SBSTH), then first-order of Brillouin Stokes line (BSL1) will be generated propagating at the opposite direction of BP. BSL1 will then travels back towards the optical coupler, where a fraction of its power is directed towards the Optical Spectrum Analyzer (OSA) as output, while rest of the power is reflected by the mirror and travels back towards the Brillouin gain medium. If the reflected BSL1 power still exceeds the SBSTH, then second-order BSL (BSL2) will be generated and propagates through the same route as BSL1. This process is repeated until BSLn does not have enough power to generate higher order BSLs.

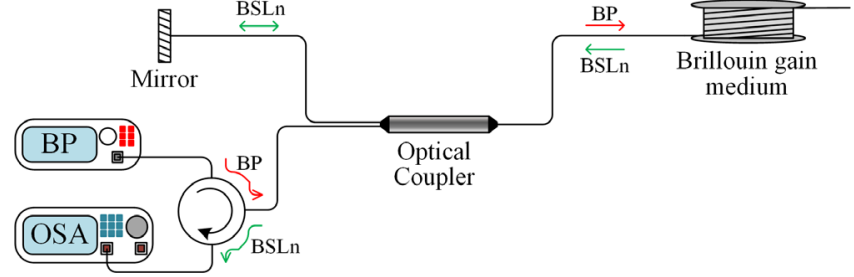

Fig. 1: Multi-wavelength Brillouin fiber laser setup.

To improve the performance of multi-wavelength Brillouin fiber laser, researchers proposes the use of additional amplifiers, namely erbium-doped fiber amplifier (EDFA) $[11,12]$ and Raman amplifier [13,14]. The usage of EDFA is known as multi-wavelength Brillouin-erbium fiber laser (MBEFL), while usage of Raman amplifier is known as multi-wavelength Brillouin-Raman fiber laser (MBRFL).

In an earlier work, Abass et. al. proposed a simple modification to conventional linear cavity MBRFL setup that extracts residual Raman pump out of the laser cavity [15]. The proposed design improves the number of BSLs compared to a conventional MBRFL, at the expense of higher Raman pump intensity required for Stimulated Brillouin Scattering (SBS) to take place.

In this paper, we investigate the effects of using the unutilized residual Raman pump to pump an erbium-doped fiber (EDF). The extracted residual Raman pump is connected to a wavelength division multiplexer (WDM) on the left-hand side of the linear cavity MBRFL. Hypothetically, this setup is able to amplify generated BSLs before propagating back towards the Brillouin gain medium. For comparison, the experiment in [15] was repeated to ensure similar performances on all the components used. Results gathered shows that our proposed setup is capable of achieving SBS at lower Raman pump intensity and is capable of producing higher number of BSLs at higher intensity of the Raman pump. 


\section{Experimental Setup}

Figure 2 depicts the experimental setup of this work. The setup consists of a Tunable Laser Source (TLS) with maximum $+6-\mathrm{dBm}$ between 1540 to $1610 \mathrm{~nm}$ which functions as Brillouin pump (BP) source. An OSA is used to measure and record the output waveform. The Raman pump unit (RPU) with 2-W maximum output power at wavlength of $1480-\mathrm{nm}$ is used as Raman source. A dispersion compensating fiber (DCF) with length of 7.1 kilometer (longest DCF available in our laboratory) functions as Brillouin and Raman gain medium. A $2 \times 23-\mathrm{dB}$ optical coupler and two Faraday mirrors (M1 and M2) form the linear laser cavity.

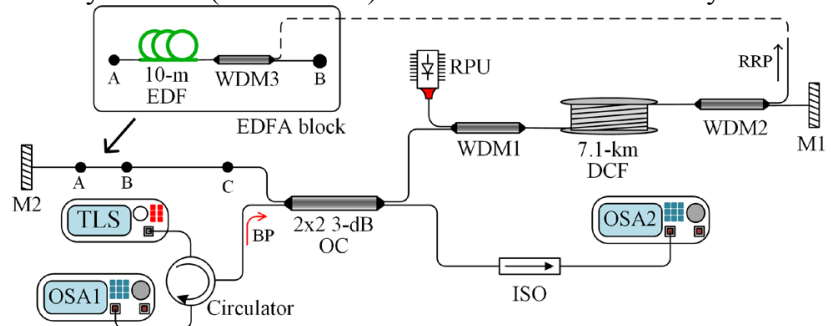

Fig. 2: Experimental setup: configuration-A, without EDFA block; configuration-B, with EDFA block. OSA: optical spectrum analyzer, TLS: tunable laser source, RPU: Raman pump unit, DCF: dispersion compensated fiber, OC: optical coupler, WDM: wavelength division multiplexer, EDF: erbium-doped fiber, M: Faraday mirror, BP: Brillouin pump, RRP residual Raman pump, ISO: isolator.

In this paper, we first repeat the experiment in [15] to ensure consistent performance of the components and devices, named configuration-A, where the erbium-doped fiber amplifier (EDFA) block is absent and extracted residual Raman pump (RPP) by WDM2 is unutilized. To reduce attenuation due to repeated coupling, configuration-A output is measured at OSA1. Configuration- $\mathrm{B}$ on the other hand has the EDFA block connected in between point $\mathrm{A}$ and $\mathrm{B}$, where the RRP is combined with oscillating BP and BSLs by WDM3 (dashed line), and output is measured at OSA2 through an isolator (ISO).

In this experiment, TLS power is fixed to $+6-\mathrm{dBm}$ and at wavelength of $1580-\mathrm{nm}$

\section{Results and Discussions}

We first investigate the gain provided by the EDFA block. This is done by adding a circulator between point $\mathrm{B}$ and $\mathrm{C}$ as shown in Figure 3. A circulator is used to ensure BSL1 does not propagates back to the DCF to generate higher order BSLs. Reflected BP and BSL1 will propagate through the circulator from port 1 to 2 , reflected by M2 and directed to OSA 3 by the circulator from port 2 to port 3 .

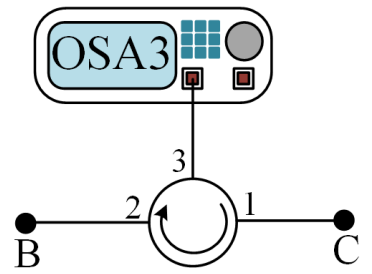

Fig. 3: Experimental setup for amplification investigation

In this investigation, the Raman pump intensity was slowly increased until BSL1 can be clearly seen at OSA3 in configurationA. Then, with the same Raman pump intensity, the output at OSA3 is measured in configuration-B. Figure 4 shows the amplification results for both configurations at Raman pump intensity of 200-mW. The result shows that the additional EDFA block is able to amplify the oscillating BP and BSL by about $15-\mathrm{dB}$ at the same Raman pump intensity. This additional gain may in turn help to achieve lower $\mathrm{SBS}_{\mathrm{TH}}$.

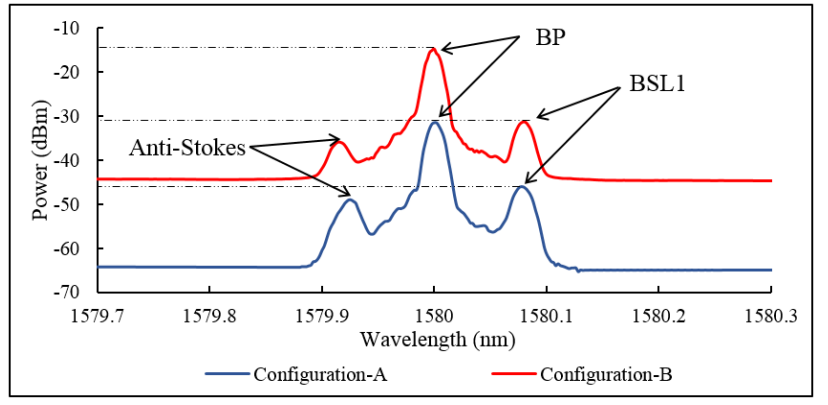

Fig. 4: Output spectrum of configuration-A and configuration-B at OSA3 with Raman pump intensity of 200-mW

Next, we compare the number of generated BSLs of configuration-A and configuration-B at varying Raman pump intensity with $25-\mathrm{mW}$ interval as shown in Figure 5. The number of BSLs for configuration-A shows consistent result reported by Abass in [15] with little discrepancies, where number of BSLs increases rapidly after Raman pump intensity of about $200-\mathrm{mW}$, and reaches its peak of 42 lines at $475-\mathrm{mW}$.

In terms of $\mathrm{SBS}_{\mathrm{TH}}$, the results in 5 show that the Raman pump required for configuration-B to generate its first BSL is as low as $50-\mathrm{mW}$, while configuration-A requires $125-\mathrm{mW}$. This shows that the additional EDFA helped in achieving lower $\mathrm{SBS}_{\mathrm{TH}}$ thanks to the low pump requirement of an EDFA.

The result in Figure 5 also shows that configuration-B produces higher BSLs at low Raman pump intensity due to the extra amplification provided by the EDFA block. The rapid increase in BSL continues until around $325-\mathrm{mW}$, where the competition between Raman and EDF amplification occurs within the laser cavity. The number of BSLs in configuration-B however increases slowly, until it generates up to 48 lines at Raman pump intensity of 600$\mathrm{mW}$. Figure 6 shows outputs comparison of configuration-A and configuration-B at its highest number of BSLs.

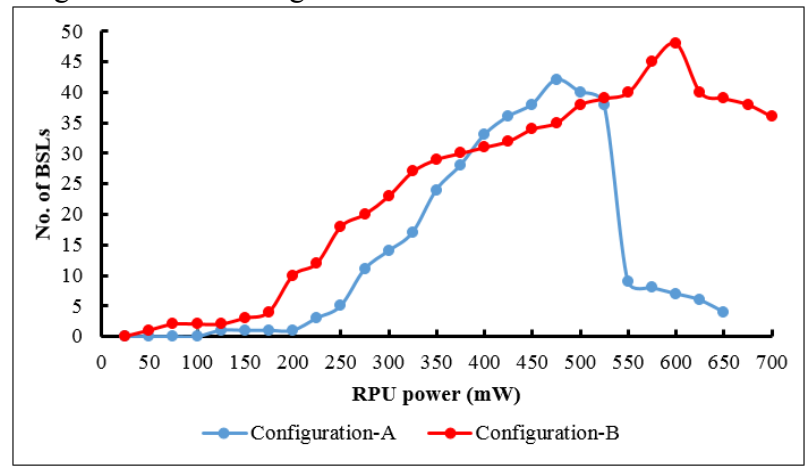

Fig. 5: Number of Brillouin Stokes lines with varying Raman pump intensity for configuration-A and configuration-B

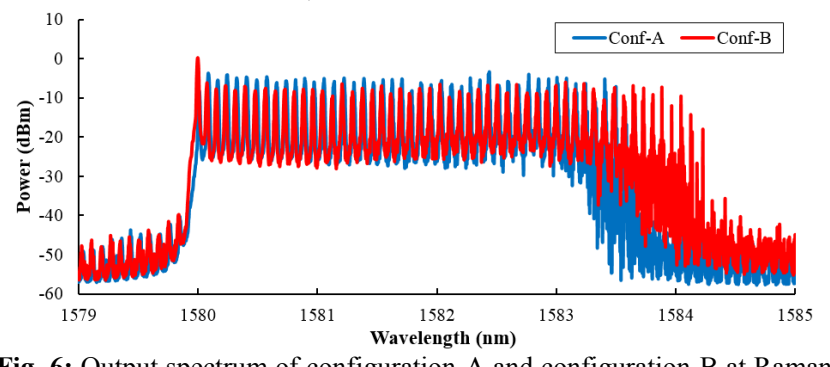

Fig. 6: Output spectrum of configuration-A and configuration-B at Raman pump intensity of 475 and $600-\mathrm{mW}$, respectively.

\section{Conclusions}

We proposed a multi-wavelength Brillouin Raman fiber laser that utilizes residual Raman pump to pump a 10-m erbium-doped fiber. Results were compared with an earlier reported work, where re- 
sidual Raman is extracted out of the cavity and left unused. The results show that the additional erbium-doped fiber amplifier is able to amplify oscillating signals by up to $15-\mathrm{dB}$. The proposed design has lower Raman pump intensity requirement for stimulated Brillouin scattering to take place and increases the number of Brillouin Stokes lines by 14\% which may beneficial for application in dense wavelength division multiplexing system.

\section{Acknowledgement}

The authors would like to thank the Universiti Tenaga Nasional for supporting this research through UNITEN BOLD grant number $10289176 / \mathrm{B} / 9 / 2017 / 38$.

\section{References}

[1] Q Li, Z X Jia, Z R Li, Y D Yang, J L Xiao, S W Chen, Y Z Huang, W P Qin and G S Qin, Broadband multi-wavelength Brillouin lasers with an operating wavelength range of 1500-1600 nm generated by four-wave mixing in a dual wavelength Brillouin fiber laser cavity, Laser Physics Letters, Vol.15, No.5, (2018).

[2] N. A. B. Ahmad, S. H. Dahlan, N. A. Cholan, H. Ahmad and Z. C. Tiu, Switchable 10, 20, and $30 \mathrm{GHz}$ region photonics-based microwave generation using thulium-doped fluoride fiber laser, Journal of the Optical Society of America B, Vol.35, No.7, (2018), pp.1603-1608.

[3] F. Zarinetchi, S. P. Smith, and S. Ezekiel, Stimulated Brillouin fiber optic laser gyroscope, Optics Letters, Vol.56, No.26, (2017), pp.7419-7426.

[4] Z. Wang, T. Wang, Q. Jia, W. Ma, Q. Su, and P. Zhang, Triple Brillouin frequency spacing multiwavelength fiber laser with double Brillouin cavities and its application in microwave signal generation, Applied Optics, Vol.16, No.4, (1999), pp.229-231.

[5] S. Saleh, N. A. Cholan ; A. H. Sulaiman ; M. A. Mahdi , Stable Multiwavelength Erbium-Doped Random Fiber Laser, IEEE Journal of Selected Topics in Quantum Electronics, Vol.24, No.3, (2018)

[6] Y. J. Song, L. Zhan, S. Hu, Q. H. Ye, Y. X. Xia, Tunable multiwavelength Brillouin-erbium fiber laser with a polarizationmaintaining fiber Sagnac loop filter, IEEE Photonics Technology Letters, Vol.16, No.9 (2004), pp.2015-2017

[7] H. Ahmad, K. Thambiratnam, A. H. Sulaiman, N. Tamchek, and S W. Harun, SOA-based quad-wavelength ring laser, Laser Physics Letter, Vol.5, No.10, (2008), pp.726-729

[8] D. R. Chen, H. Fua, H. Oua and S. Qin, Wavelength-spacing continuously tunable multi-wavelength SOA-fiber ring laser based on Mach-Zehnder interferometer, Optics \& Laser Technology, Vol.40, No.2, (2008), pp.278-281.

[9] M. R. Shirazi, S. W. Harun and H. Ahmad, Multi-wavelength Brillouin Raman erbium-doped fiber laser generation in a linear cavity, Journal of Optics, Vol.16, No.3, (2014), pp.278-281

[10] A. K. Abass, M. H. Al-Mansoori, M. Z. Jamaludin, F. Abdullah, and T. F. Al-Mashhadani, Characteristics of multiwavelength Lband Brillouin-Raman fiber laser under forward and backward pumped environment, Applied Optics, Vol.52, No.16, (2013), pp.3764-3769

[11] G. J. Cowle and D. Y. Stepanov, Hybrid Brillouin/erbium fiber laser, Optics Letters, Vol.21, No.16, (1996), pp.1250-1252

[12] N. A. B. Ahmad, N. A. Cholan and S. H. Dahlan, Numerical analysis of signal recycling in multiwavelength Brillouin-erbium fiber laser, 2017 Conference on Lasers and Electro-Optics Pacific Rim (CLEO-PR), Vol.2017, No.5, (2017), pp.1-3

[13] B. Min, P. Kim and N. Park, Flat amplitude equal spacing 798 channel Rayleigh-assisted Brillouin/Raman multiwavelength comb generation in dispersion compensating fiber, IEEE Photonics Technology Letters, Vol.13, No.12, (2001), pp.1352-1354

[14] G. Mamdoohi, A. R. Sarmani, M. H. A. Bakar and M. A. Mahdi, Switchable Multiwavelength Brillouin-Raman Fiber Laser Utilizing an Enhanced Nonlinear Amplifying Fiber Loop Design, IEEE Photonics Journal, Vol.10, No.2, (2018), pp.1-11

[15] A. K. Abass, M. H. Al-Mansoori, M. Z. Jamaludin, F. Abdullah, T. F. Al-Mashhadani and M. H. Ali, Enhancing performance of multiwavelength Brillouin-Raman fiber laser by capturing residual pump power, Applied Optics, Vol.53, No.23, (2014), pp.5187-5192 